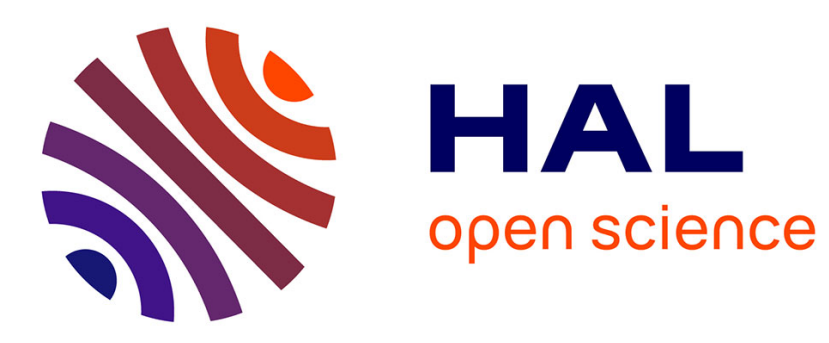

\title{
Silicon Probe Measurement and Characterization in Sub-THz Range
}

Haotian Zhu, Jules Gauthier, Ke Wu

\section{To cite this version:}

Haotian Zhu, Jules Gauthier, Ke Wu. Silicon Probe Measurement and Characterization in SubTHz Range. IEEE Transactions on Terahertz Science and Technology, 2020, 10 (6), pp.606-616. 10.1109/TTHZ.2020.3013802 . hal-03094246

\section{HAL Id: hal-03094246 https://hal.sorbonne-universite.fr/hal-03094246}

Submitted on 4 Jan 2021

HAL is a multi-disciplinary open access archive for the deposit and dissemination of scientific research documents, whether they are published or not. The documents may come from teaching and research institutions in France or abroad, or from public or private research centers.
L'archive ouverte pluridisciplinaire HAL, est destinée au dépôt et à la diffusion de documents scientifiques de niveau recherche, publiés ou non, émanant des établissements d'enseignement et de recherche français ou étrangers, des laboratoires publics ou privés. 


\title{
Silicon Probe Measurement and Characterization in Sub-THz Range
}

\author{
Haotian Zhu, Member, IEEE, Jules Gauthier, and Ke Wu, Fellow, IEEE
}

\begin{abstract}
We propose, investigate and demonstrate a pure and simple dielectric probe operating in the sub- $\mathrm{THz}$ range with an example band of interest 190-220 GHz. High-resistivity silicon technology is deployed for this non-TEM mode probe development as it presents a viable low-loss material. A T-shape alignment dowel was used to integrate the silicon probe with the waveguide block. The probe can be used to measure and characterize the low permittivity-based dielectric waveguide transmission line and its building components free from the use of any transition structures and TEM-mode probes. For demonstration, the silicon probe was used to measure a substrate integrated image guide and its components in the range of 190-220 GHz. A substrate integrated image guide (SIIG) based TRL calibration kit was developed, and a two-tier TRL calibration was used to characterize the SIIG.
\end{abstract}

Index Terms-Dielectric transmission line, laser micromachining, probe technology, silicon assembling, substrate integrated image guide (SIIG).

\section{INTRODUCTION}

$\mathrm{T}$ HE sub-terahertz (sub-THz) technology - usually referring to technology operating in the electromagnetic spectrum over the $100-500 \mathrm{GHz}$ - has widely been used in radio astronomy, imaging, radar, remote sensing and short-range communication systems [1]-[4]. A successful deployment of sub- $\mathrm{THz}$ technologies and systems requires accurate measurements and characterization of related transceiver building blocks, such as front-end components and circuits, prior to their design and development. These parts may be designed and fabricated through planar and non-planar metallic, dielectric and metallo-dielectric hybrid topologies. The dielectric waveguide is a good candidate for $\mathrm{THz}$ circuit and antenna developments and applications thanks to its low-loss, low-cost and easy planar integration. There are several planar dielectric waveguides in sub-THz range, such as dielectric rigid waveguide [5], dielectric microstrip line (DML) [6], [7], substrate integrated image guide (SIIG) [8], U-silicon-on-glass waveguide [9], etc. The fundamental propagation mode for the dielectric waveguide is the $E_{11}^{x}$ mode or $E_{11}^{y}$ mode, according to its excitation polarization [8], [9], which has a similar E-field distribution to the $T E_{10}$ for the metal rectangular waveguide [7]. However, measuring a dielectric waveguide and its components

This Manuscript received XXXX, 2020; revised XXXX, XXXX; accepted XXXX, XXXX. This work was supported by the NSERC-Huawei Industrial Research Chair Program.

H.-T. Zhu was with the Poly-Grames research center, Ecole Polytechnique de Montreal, Montreal, QC H3T1J4, Canada. He is now with the LERMA, Observatoire de Paris, Sorbonne Université, Université PSL, CNRS, Paris 75014, France (e-mail: zhuhaotian_2007@126.com).

J. Gauthier and K. Wu are with the Poly-Grames research center, Ecole Polytechnique de Montreal, Montreal, QC H3T1J4, Canada (e-mail: jules.gauthier@polymtl.ca; ke.wu@polymtl.ca).

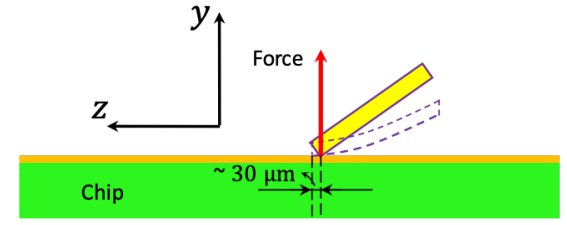

Fig. 1. Traditional GGB GSG probe; the probe bends in measurement and 30 $\mu \mathrm{m}$ shift in z-axis, (side view).

could be a daunting task as the resulting measurement set up may not be compatible with current measurement standards. There are two major methods to test the dielectric waveguide, namely the non-contact and contact methods. For the non-contact method, a quasi-optical circuit design is always required [5]-[6]. Generally, the final bulky quasi-optical circuit needs a precise adjustment [10]. For the contact method, one simple strategy is to insert the dielectric waveguide into a rectangular waveguide, which causes inconvenience for taking measurements, especially at high frequencies [7]. Another alternative method is using a probe to carry out the test.

A probe station is a traditional commercial method to measure RF circuits. Probes are known as a very important RF measurement tool in characterizing the performances of active/passive components, circuits and systems; quite often parameter extractions and performance evaluations are conducted in this process. Generally, the development of probes becomes more difficult as frequency increases. The reason is related to the mechanical precision and electrical reliability as well as transmission loss, which are all critical for sub-THz and beyond. Recently, attempts have been made for accurate measurements and characterization of sub- $\mathrm{THz}$ components and circuits. Based on coaxial cable techniques, industrial products can provide a high performance probe at up to $1.1 \mathrm{THz}$. For example, the company GGB Inc. provides commercial CPW-based THz probe [11]. Fig. 1 shows the side view of a metal-based GGB ground-signal-ground (GSG) probe. Once touching the surface of a circuit, the probe will reshape its structure and extend approximate $30 \mu \mathrm{m}$ under a simple pressing. Nevertheless, such standard GSG probes are designed through a CPW structure with TEM mode input conditions. Moreover, in 2011, the group of Robert M. Weikle, II and N. Scott Barker proposed a set of performance-enhanced micro-machined probes for sub-THz applications [12], [13].

Generally, two transitions are needed to measure a dielectric waveguide operating over the sub-THz band, where one transition is designed for coupling the $T E_{10}$ mode of a frequency extender output to the TEM mode of a GSG probe and the other transition is set for coupling the TEM mode of a GSG probe to the $E_{11}^{x}$ or $E_{11}^{y}$ mode of the dielectric waveguide [14]-[16]. Therefore, it is inconvenient to measure the dielectric waveguide or its constituent components. 


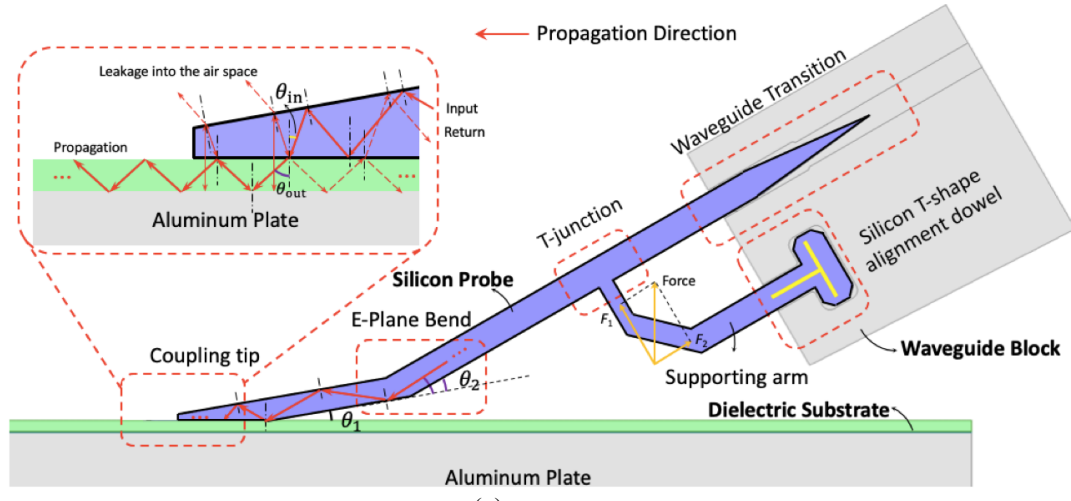

(a)

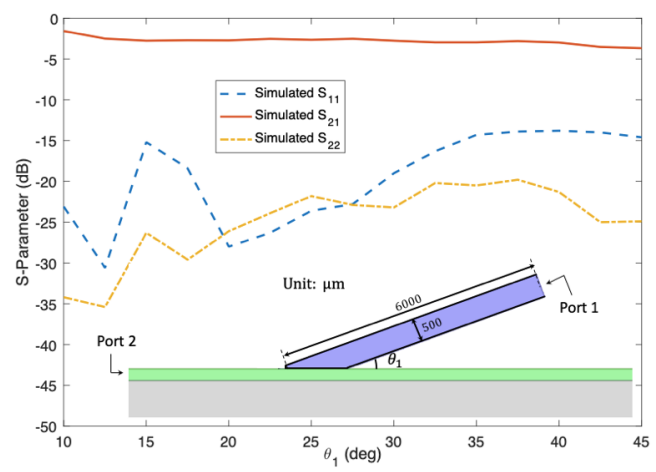

(b)

Fig. 2. (a) Silicon-based dielectric probe structure and its working principle at $200 \mathrm{GHz}$ in side view $\left(\theta_{1}=10^{\circ}\right.$ and $\left.\theta_{2}=20^{\circ}\right)$. (b) Coupling tip structure and parameter study of the $\theta_{1}$ at $200 \mathrm{GHz}$.

In this work, a highly original scheme of dielectric probe is proposed and demonstrated, which can be used to measure and characterize sub- $\mathrm{THz}$ dielectric waveguides and components. Without transferring to the TEM mode or intermediate modal transition, the $T E_{10}$ mode in the metal rectangular waveguide can be converted to the $E_{11}^{y}$ mode in the dielectric waveguide directly. Compared with non-contact methods [5], [6], the dielectric probe method does not need to conduct a stringent quasi-optical circuit alignment. To the authors' knowledge, this is the first time the concept of dielectric probe is demonstrated. In this work, silicon techniques are used as examples for our demonstration as they are popular and low-loss. The substrate integrated image guide (SIIG) is a popular dielectric waveguide in the sub- $\mathrm{THz}$ range [8]. The advantages of SIIG are its low-cost and easy PCB fabrication and integration. In this paper, the silicon probe is used to measure the SIIG and its components.

\section{CONCEPT OF Silicon Probe}

In this work, a dielectric probe concept is devised, designed and demonstrated for probing measurements and characterization over the low-end of sub-THz, which can be extended to the high-end of the sub-THz range, depending on the topology and applications of a dielectric probe of choice. As shown in Fig. 2(a), a detailed graphic illustration is presented for possible measurement and characterization scenarios envisioned in this work. A block of waveguide is connected to a sub-THz source. A silicon-based dielectric probe (blue part) is set in contact with a dielectric substrate (green part).

The proposed probe consists of a coupling tip, $E$-plane bend, $\mathrm{T}$-junction, and waveguide transition. With the wave number marked in Fig. 2(a), the coupling tip can couple the EM energy from the silicon-based dielectric waveguide (SiDW) into another dielectric waveguide, which is the SIIG in this paper. The principle of coupling can approximatively be explained using geometric optics. In the demonstration model, the cross-sectional size of the SiDW of the coupling tip is 0.915 $\mathrm{mm} \times 0.500 \mathrm{~mm}$. The main material of the SIIG is RO3035 PCB board. The cross-sectional size of the SIIG is $1.1 \mathrm{~mm} \times$ $0.254 \mathrm{~mm}$. The permittivity of RO3035 and high-resistivity silicon in $140-220 \mathrm{GHz}$ are 3.75 and 11.86 , respectively. The values were measured using our own sub- $\mathrm{THz}$ substrate permittivity measurement platform [17].
As shown in Fig. 2(a), before being guided into the SIIG, the EM wave undergoes several reflections along the E-plane bend and coupling tip. Most of those reflections are total reflections. Finally, when the angle of incidence $\left(\theta_{\text {in }}\right)$ into the silicon probe is less than the critical angle between the SiDW and free space $\left(\theta_{c 0}=19.2^{\circ}\right.$ at $\left.200 \mathrm{GHz}\right)$, the EM can also be leaked out of the probe and transferred into free space. The behavior of $\theta_{\text {in }}$ is described using the following geometric relationship

$$
\theta_{\text {in }}=90^{\circ}-n \cdot \theta_{1}-\theta_{2}, \quad n=1,3,5 \cdots
$$

Snell's law can be used to describe the refraction occurring on the boundary between the silicon probe and the SIIG in the coupling tip part

$$
\frac{\sin \theta_{\text {in }}}{\sin \theta_{\text {out }}}=\frac{\beta_{\text {SIIG }}}{\beta_{\text {SiDW }}}=\frac{\beta_{\text {SIIG }} / k_{0}}{\beta_{\text {SiDW }} / k_{0}}
$$

where $\theta_{\text {out }}, \beta_{\text {SIIG }} / k_{0}$ and $\beta_{\text {SiDW }} / k_{0}$ are the angles of refraction in the SIIG, SIIG normalized phase constant and SiDW normalized phase constant, respectively. According to simulated results at $200 \mathrm{GHz}, \beta_{\text {SIIG }} / k_{0}$ and $\beta_{\text {SiDW }} / k_{0}$ are 1.42 and 3.04, respectively. When the incident angle into the silicon zone is less than the critical angle $\left(\theta_{c 1}=27.8^{\circ}\right.$ at $\left.200 \mathrm{GHz}\right)$, the wave propagates into the SIIG. To satisfy this requirement, $n$ is equal to $5, \theta_{\text {in }}$ is equal to $20^{\circ}$ and $\theta_{\text {out }}$ is equal to $47^{\circ}$ at $200 \mathrm{GHz}$. On the boundary between the substrate and the ground, which is made of an aluminum plate, the EM is subject to the total reflection. On the boundary between the substrate and free space, the EM also undergoes total reflection. Since $\theta_{\text {out }}$ is larger than the critical angle between the SIIG and free space $\left(\theta_{c 2}=44.8^{\circ}\right.$ at $\left.200 \mathrm{GHz}\right)$, the EM wave propagating in the SIIG is along the boundary between the free space and the substrate as a forced surface wave. The propagation mode of the SIIG is the fundamental $E_{11}^{y}$ mode [8]. For any dielectric probe design, the critical issue of the design is to choose a suitable material according to the two critical angles $\theta_{c 1}$ and $\theta_{c 2}$. The design emphasis is then on the choice of geometric sizes or dimensions of the dielectric waveguide for the probe to satisfy (1) and (2).

Considering the equivalent permittivity or $\beta_{\mathrm{SiDW}} / k_{0}$ changes in the coupling tip, the actual optical model of the 


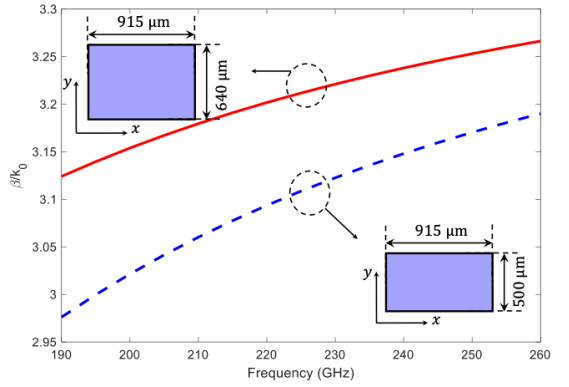

(a)

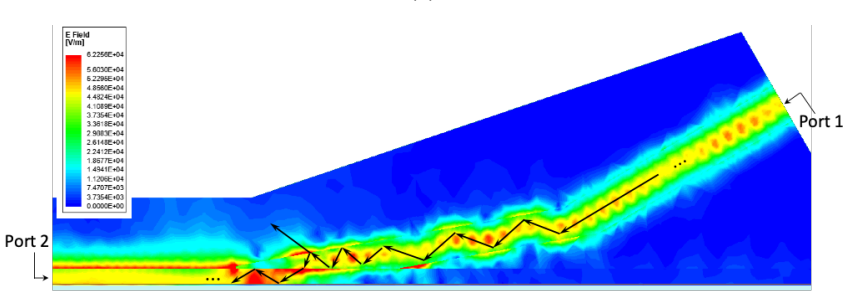

(c)

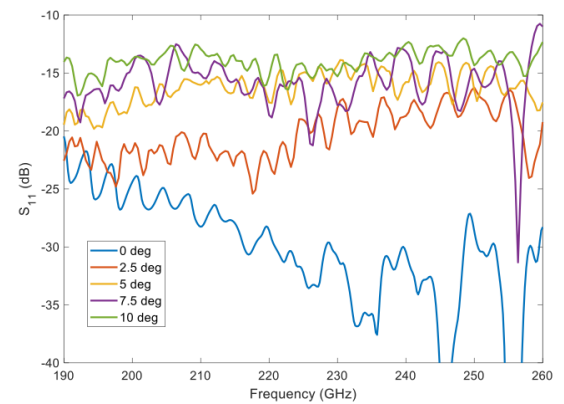

(e)

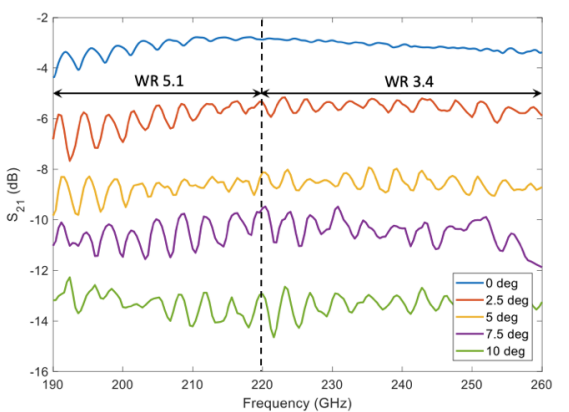

(f)

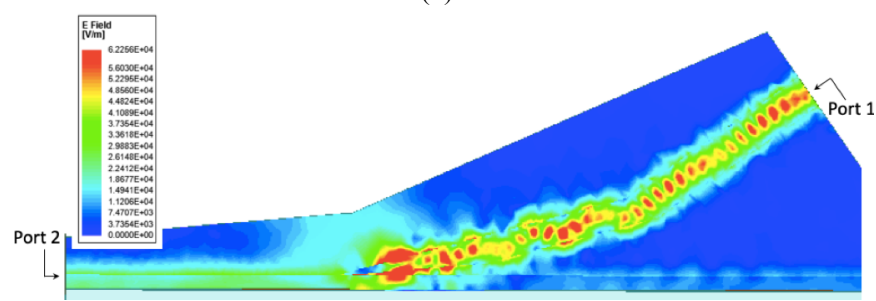

(d)

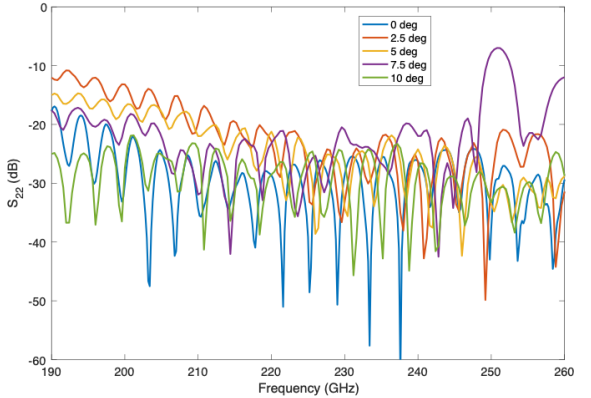

(g)

Fig. 3. Coupling tip and $E$-plane bend parts. (a) Normalized phase constant of SiDW. (b) Model in side view. (c) $E$-field complex magnitude with $\theta_{3}=0^{\circ}$ and (c) $\theta_{3}=5^{\circ}$ at $200 \mathrm{GHz}$. (e) Simulated $\mathrm{S}_{11}$, (f) simulated $\mathrm{S}_{21}$ and (g) simulated $\mathrm{S}_{22}$ with $\theta_{3}$ ranges from $0^{\circ}$ to $10^{\circ}$.

silicon probe is more complex. Commercial full-wave electromagnetic simulation software Ansoft HFSS simulates the coupling performance of the probe and SIIG. The relationship between the performance of the coupling tip and the inclination angle $\left(\theta_{1}\right)$ is shown in Fig. 2(b). The length of the silicon probe is fixed to be $6 \mathrm{~mm}$ and $\theta_{1}$ changes from $10^{\circ}$ to $45^{\circ}$ with a step of $2.5^{\circ}$. The coupling is stronger and return loss is better at lower inclination angle. However, if $\theta_{1}$ is less than $10^{\circ}$, the contact area will be much larger and the silicon probe will be much longer. Therefore, $\theta_{1}=10^{\circ}$ is chosen for the final design.

The cross-sectional interface sizes of the $E$-plane bend are $0.915 \mathrm{~mm} \times 0.50 \mathrm{~mm}$ and $0.915 \mathrm{~mm} \times 0.64 \mathrm{~mm}$, and their normalized phase constants are shown in Fig. 3(a). The coupling tip and the $E$-plane bend model of the silicon probe are shown in Fig. 3(b). As shown in Fig. 3(c), the simulated performance is similar to the analyzed results using geometric optics. In the experiments, there will be an intersection angle $\theta_{3}$ between the tip and the surface of a dielectric transmission line under test. The simulated performance of the coupling tip and E-plane bend part with $\theta_{3}=5^{\circ}$ is shown in Fig. 3(d). As shown in Fig. 3(b), the EM energy radiates into the free space and leaks into the reserve propagation direction of the SIIG when the intersection angle exists. The return loss of port 1, the insertion loss and return loss of port 2 of the structure in Fig. $3(\mathrm{~b})$, with $\theta_{3}$ ranging from $0^{\circ}$ to $10^{\circ}$ are shown in Figs. 3(e), (f)

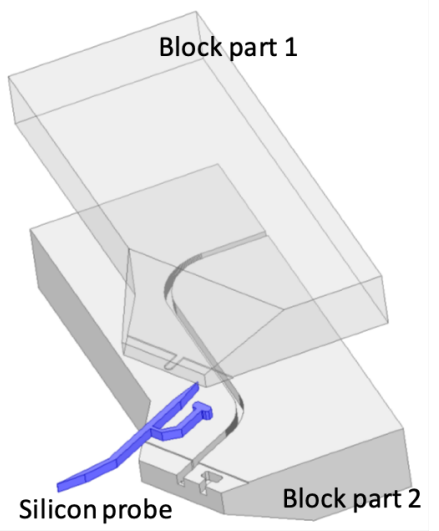

(a)

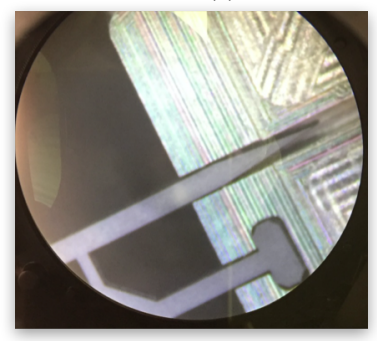

(c)

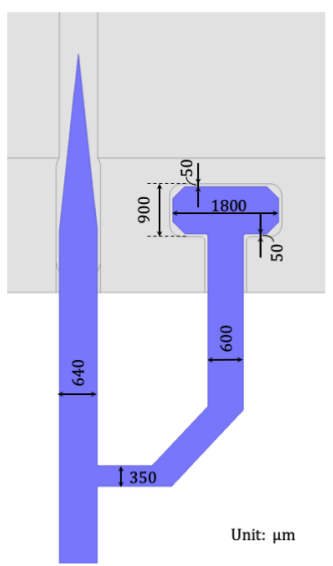

(b)

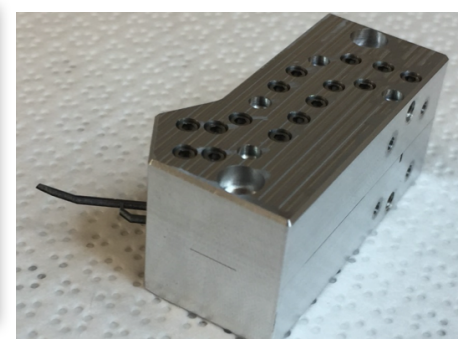

(d)
Fig. 4. Assembly technology. (a) Exploded view of the assembling structure of silicon probe block, (b) details of the silicon T-shape alignment dowel, (c) picture of the silicon T-shape alignment dowel, and (d) assembled silicon probe block. 
and $(\mathrm{g})$, respectively. The insertion loss is almost less than $4 \mathrm{~dB}$ at $\theta_{3}=0^{\circ}$ in the frequency band of $190-260 \mathrm{GHz}$. The return loss of port 1 at $\theta_{3}=0^{\circ}$ is better than $20 \mathrm{~dB}$ over the range of $190-260 \mathrm{GHz}$ (WR 5.1 band). The insertion loss increases when $\theta_{3}$ increases.

The cross-sectional interface sizes of the silicon-based probe waveguide and the standard WR 5.1 rectangular waveguide are $0.915 \mathrm{~mm} \times 0.64 \mathrm{~mm}$ and $1.295 \mathrm{~mm} \times 0.648 \mathrm{~mm}$, respectively. To assemble the SiDW into the waveguide block, a special waveguide transition is designed, as shown in Fig. 4. The silicon probe is packaged by two waveguide block parts. A $\mathrm{T}$-junction provides an arm in support of the contacting force during measurements, as described in Fig. 2(a). The resolution of contacting force is $F_{1}$ and $F_{2} . F_{1}$ supports the main silicon probe beam via the T-junction, and $F_{2}$ can shift the silicon T-shape alignment dowel along the groove of the waveguide block slightly. The maximum shift caused by $F_{2}$ is $50 \mu \mathrm{m}$ along the silicon T-shape alignment dowel, as marked in Fig. 4(b). Meanwhile, the isolation of the T-junction is more than 25 $\mathrm{dB}$ and the insertion loss of the T-junction is less than $0.5 \mathrm{~dB}$, which almost barely affects the transmission performance. The working principle of the T-junction is similar to the crossover in [6]. The $E$-plane $20^{\circ}$ bend structure is used for reducing the length of the silicon probe. The insertion loss of the $E$-plane bend is less than $1.4 \mathrm{~dB}$. After laser cutting, the silicon-based probe can be released from a $0.915 \mathrm{~mm}$-thickness high-resistivity silicon wafer. The laser beamwidth is around $90 \mu \mathrm{m}$. The aluminum housing waveguide block parts are fabricated by our own CNC technology in the Poly-Grames Research Center. Finally, using the silicon T-shape alignment dowel, the housing block and the silicon-based probe were neatly assembled, as shown in Figs. 4(c) and (d). More simulated results of the silicon probe and SIIG structure can be found in Part C, Section III.

\section{SIIG AND ITS COMPONENTS DESIGN}

As shown in Fig. 6, the SIIG structure is made of two parts: one is the $3.175 \mathrm{~mm}$ thick single-side polished aluminum plate, which provides an excellent ground for the SIIG, and the other one is the $0.254 \mathrm{~mm}$ thick RO3035 PCB board. The width of the SIIG is $1.1 \mathrm{~mm}$. For the PCB board, one-layer cladding copper layer was removed. The laser micromachining equipment in the Poly-Grames Research Center was used to create the designed pattern on the PCB board. An epoxy adhesive was used to bond the PCB board and the aluminum plate. The thickness of the epoxy adhesive is $\sim 5 \mu \mathrm{m}$ which is far less than the working wavelength of $1.5 \mathrm{~mm}$.

\section{A. Modal Analysis and Coupling Characteristics}

Fig. 5(a) shows the normalized phase constant $\left(\beta / k_{0}\right)$ for the first three modes of the SIIG, which are $E_{11}^{y}, E_{21}^{y} E_{31}^{y}$ modes. It is difficult to excite the odd symmetry $E_{21}^{y}$ modes by the even symmetry $T E_{10}$ mode in the WR 5.1 waveguide. Considering the attenuation loss of the SIIG for the $E_{11}^{y}$ mode, a low attenuation loss starts from $190 \mathrm{GHz}$, as shown in Fig. 5(b). Considering the $E_{31}^{y}$ mode appearing at $237 \mathrm{GHz}$, the SIIG can support the $E_{11}^{y}$ mode propagation from 190-237 GHz. However, $220 \mathrm{GHz}$ is the frequency point which is the highest

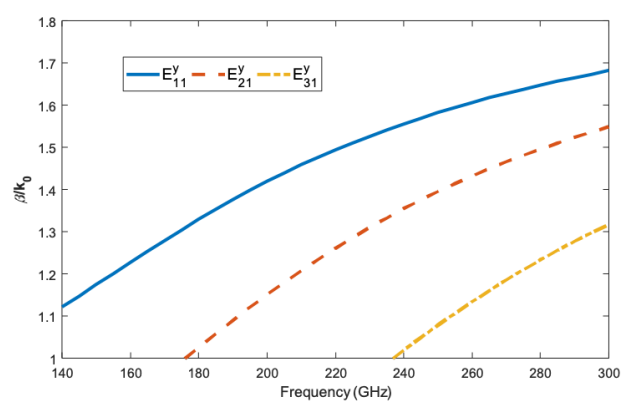

(a)

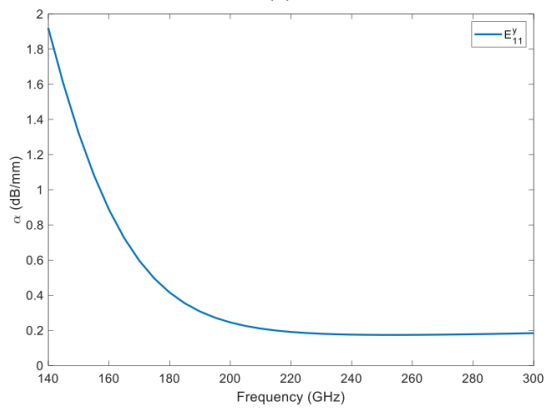

(b)

Fig. 5. Propagation constant of SIIG (a) normalized phase constant and (b) attenuation constant.

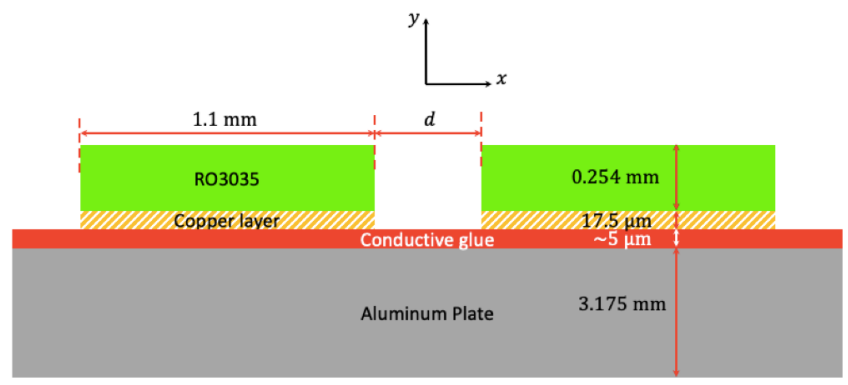

Fig. 6. Two SIIG cross-section views (not to scale).
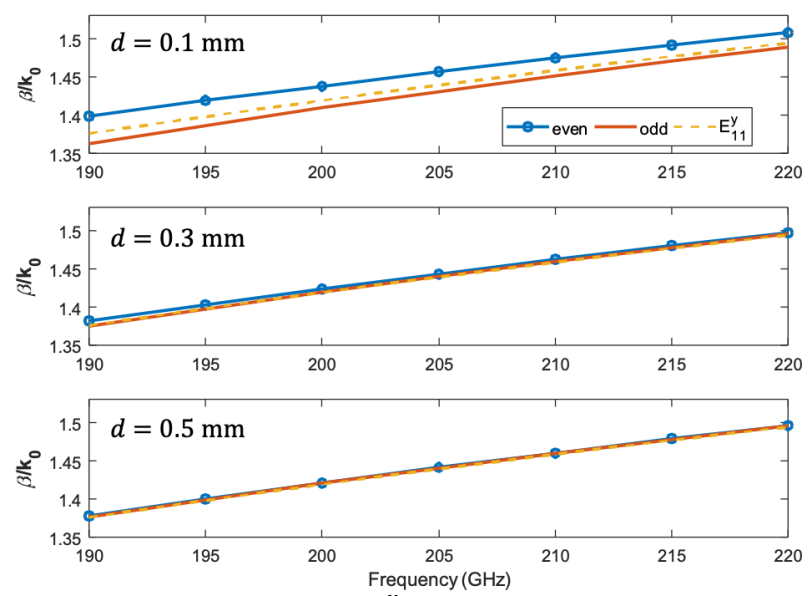

Fig. 7. Normalized dispersions of $E_{11}^{y}$ mode of single SIIG and even-odd modes of two adjacent SIIGs.

frequency of the WR 5.1 band (140-220 GHz) and the lowest frequency of the WR3.4 band (220-330 GHz), as shown in Fig. 3(f). Therefore, to simplify the intended experimental validation, the designed band is $190-220 \mathrm{GHz}$.

The structure of two adjacent SIIGs is shown in Fig. 6. To analyze the coupling of the proposed SIIG, two adjacent SIIGs are compared with a single SIIG. Fig. 7 presents the normalized phase constants of the $E_{11}^{y}$ mode of the SIIG and the even-odd 


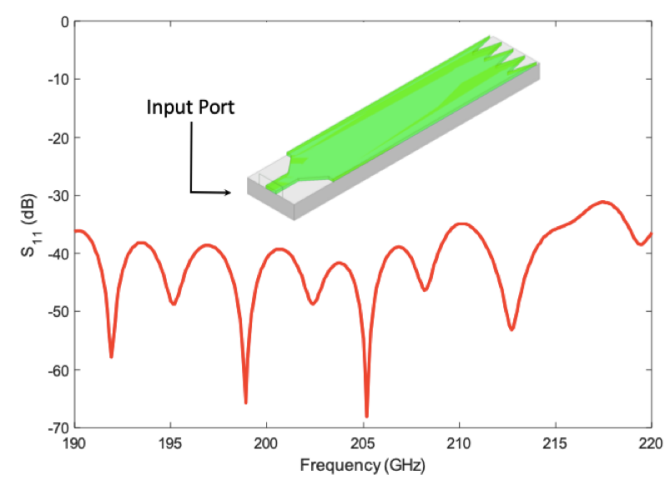

Fig. 8 SIIG $2 \mathrm{D}$ absorber and its $\mathrm{S}_{11}$.

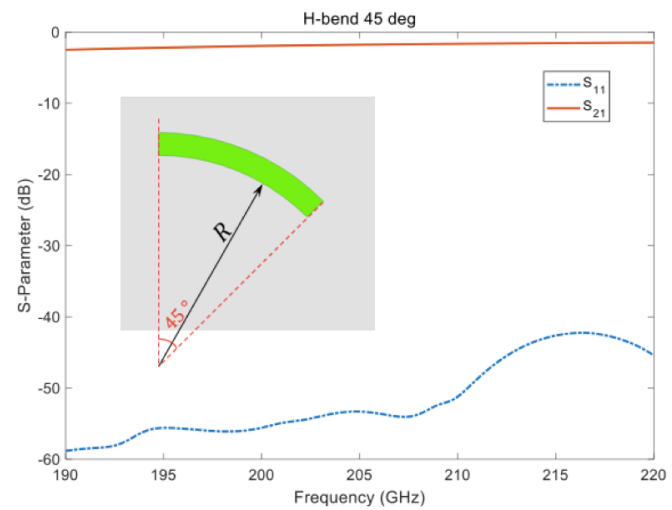

Fig. 9. SIIG-based $H$-bend S-parameter $(R=10.16 \mathrm{~mm})$

modes of two SIIGs spaced with different distances $(d$ from 0.1 to $0.5 \mathrm{~mm}$ ). As $d$ increases, the normalized phase constants of the three modes become closer, and when $d \geq 0.5 \mathrm{~mm}$, they almost become the same line. For demonstration, $d$ of the two SIIGs is set to $2.54 \mathrm{~mm}(100 \mathrm{mil})$ in this work.

\section{B. Absorber and H-plane Bend of SIIG}

The designed 2D absorber of the SIIG is shown in Fig. 8. The saw tooth and the H-plane taper are used to guide the EM waves to the free space. The return loss is less than $30 \mathrm{~dB}$. To construct SIIG-based components, a SIIG-based bend is necessary. A SIIG-based $H$-plane bend with a $10.16 \mathrm{~mm}(400$ mil) radius was designed. As shown in Fig. 9, it can be seen that the insertion loss and return loss of the $H$-bend are better than $2.7 \mathrm{~dB}$ and $40 \mathrm{~dB}$, respectively.

\section{Probe Performance and Short Circuit}

The contact probe model of the SIIG and the silicon probe in the housing waveguide block are shown in Fig. 10(a). This model is the error box for TRL calibration. Fig. 10(a) shows the S-parameters. The return losses of the SIIG port for the $E_{11}^{y}$ mode and WR5.1 waveguide port are both better than $17 \mathrm{~dB}$. The insertion losses for the $E_{11}^{y}$ mode and $E_{21}^{y}$ mode of the one-arm silicon probe and SIIG are approximately $5.8 \mathrm{~dB}$ and $61 \mathrm{~dB}$, respectively. Therefore, the $E_{21}^{y}$ mode can be neglected. The details of the insertion loss of the one-arm silicon probe and SIIG are shown in Table I. When the SIIG input port (port 2) is shorted, the return loss of the port 1 is approximately $10.8 \mathrm{~dB}$, as shown in Fig. 10(a). The reason is that part of the EM energy propagates along the SIIG to the absorber after being reflected by the short circuit of the SIIG input port. Fig. 10(b) shows the

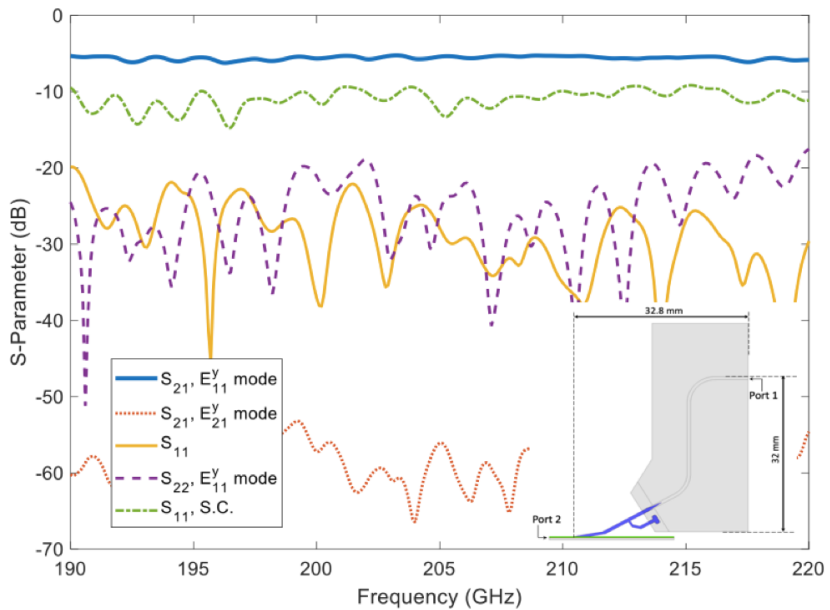

(a)

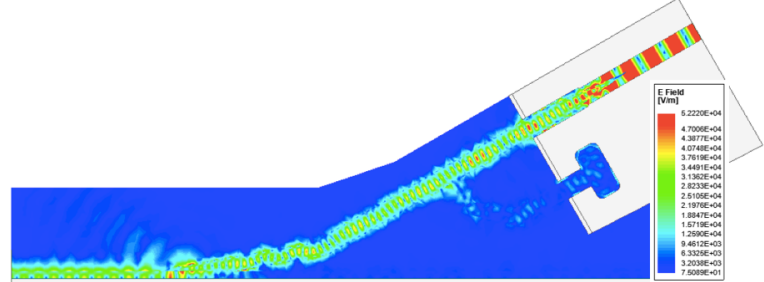

(b)

Fig. 10. Error box (a) Side view of one-arm silicon probe and SIIG and simulated S-parameter of one-arm silicon probe and SIIG. (b) Simulated $E$-field magnitude distribution of the error box without WR $5.1 E$-bend at $200 \mathrm{GHz}\left(\theta_{3}=0^{\circ}\right)$.

\begin{tabular}{|c|c|} 
TABLE I LOSSES IN SILICON PROBE AT $200 \mathrm{GHz}$ \\
\hline Structure for $E_{11}^{y}$ mode & Value $(\mathrm{dB})$ \\
\hline Waveguide block & 0.8 \\
\hline $\begin{array}{c}\text { WR5.1 and SiDW } \\
\text { transition }\end{array}$ & 1.6 \\
\hline$E$-plane bend & 1.4 \\
\hline Coupling tip & 2 \\
\hline Total & 5.8 \\
\hline
\end{tabular}

simulated $E$-field magnitude distribution of the contact model without WR 5.1 E-bend.

\section{SIIG-based Coupler Design}

The top view of the SIIG-based coupler structure is shown in Fig. 11, which is similar to the DML-based coupler [6]. As shown in Fig. 11, the insertion loss between the input port 1 and the through port 3 is smaller than $2.18 \mathrm{~dB}$. The coupling factor is around $-14.42 \mathrm{~dB}$. The return loss of port 1 is larger than 23.35 $\mathrm{dB}$. The isolation between port 1 and port 4 is larger than 24.46 $\mathrm{dB}$.

\section{E. SIIG Layout Design}

The final layout of the SIIG and its components' design are shown in Fig. 12. The layout consists of alignment graduation markings, reference, coupler, and thru, reflect and line (TRL) kit, absorbers, and two adjacent SIIGs. The coupler consists of two reference SIIGs with a $45^{\circ}$ angle. Two alignment pins were used to mount the RO 3035 PCB layer onto the aluminum plate. Alignment graduation markings were plotted on the front side of the SIIG by laser micromachining, as shown in Fig. 12. The fabricated line width is $48.82 \pm 4.35 \mu \mathrm{m}$. The TRL kit consists 


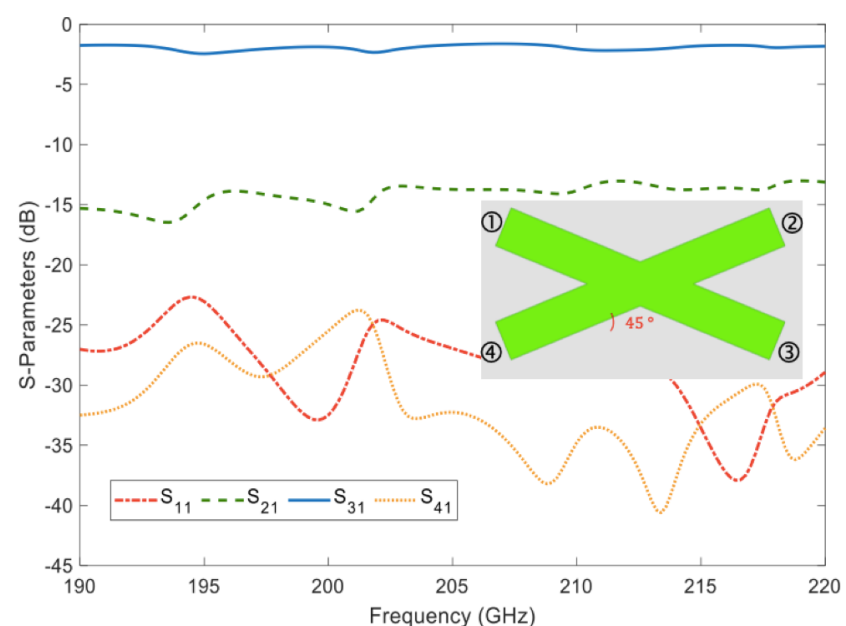

Fig. 11. SIIG-based coupler: top view and simulated S-parameter and phase difference between coupler port and through port.

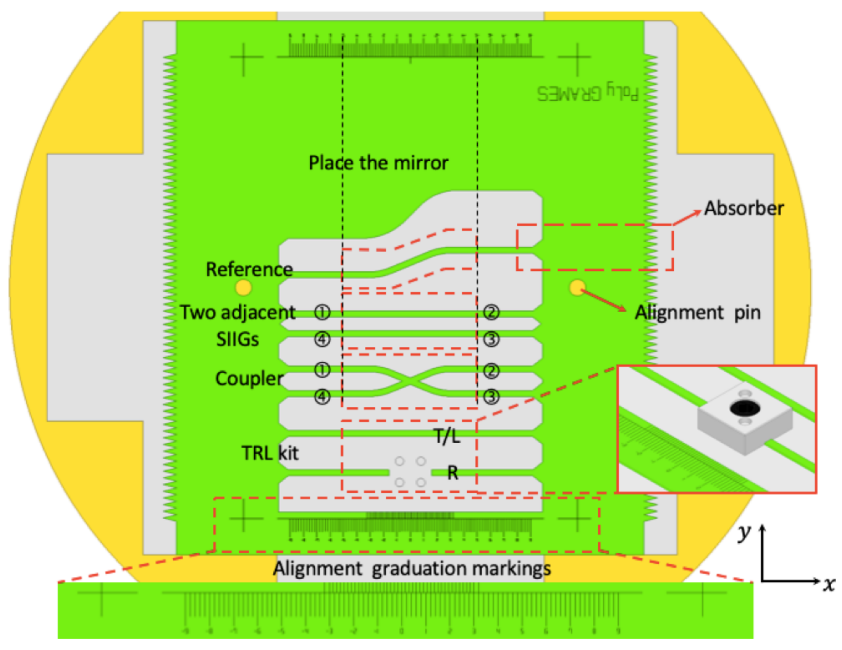

Fig. 12. Details of SIIG layout, top view.

of two parts: the upper is for thru and line $(\mathrm{T} / \mathrm{L})$, and the lower is for reflection (R) [18]. As shown in Fig. 15(b), the lengths of T and $\mathrm{L}$ are 360 mil and 375 mil, respectively. The phase delay between $\mathrm{T}$ and $\mathrm{L}$ is $129.5^{\circ}$ at $200 \mathrm{GHz}$. As shown in the inset in Fig. 12, an aluminum box $(8.13 \mathrm{~mm} \times 8.08 \mathrm{~mm} \times 3.05 \mathrm{~mm}$ or 320 mil $\times 318$ mil $\times 120$ mil) was fabricated to realize the short circuit reflection. The distance between the silicon probe and the sidewall of the aluminum box is $180 \mathrm{mil}$, as shown in Fig. 15(c). Moreover, two dowel pins are used for alignment, and one screw is used for mounting the aluminum box onto the aluminum plate.

\section{Probe Characterization and SiIG Measurement}

Fig. 13 shows the whole silicon probe measurement system. In this set-up, two VDI WR 5.1 band extenders and one Keysight PNA-X N5274A vector network analyzer (VNA) are used to provide the sources and vector detectors in the measurement system over 190-220 GHz. For each probe arm, one CERNEX 1-inch straight waveguide and $180^{\circ} \mathrm{E}$-bend WR5.1 band waveguide are used to connect the VDI WR5.1 extender and the waveguide-based probe block. The Suss microtech semi-automatic probing station is used to place the VDI WR5.1 extenders via two home-made fixtures, as shown

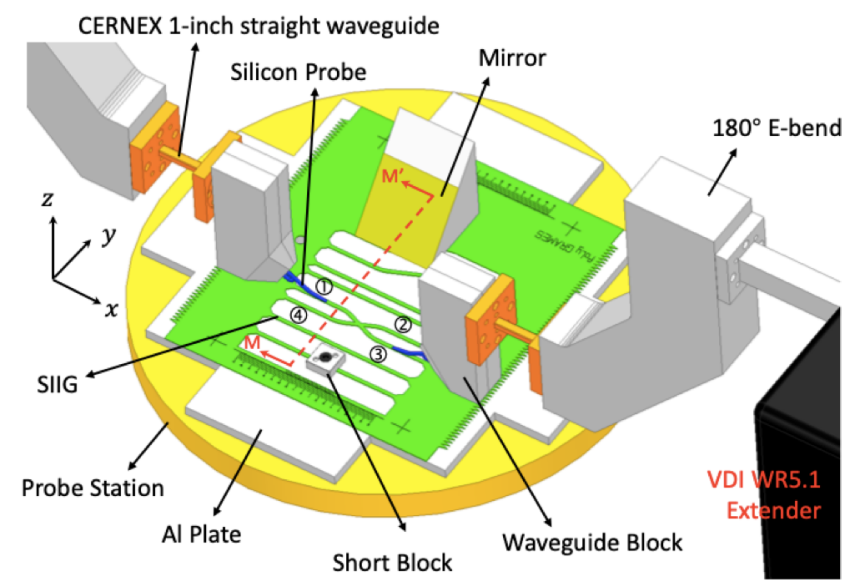

Fig. 13. Silicon probe measurement system in 3D view.

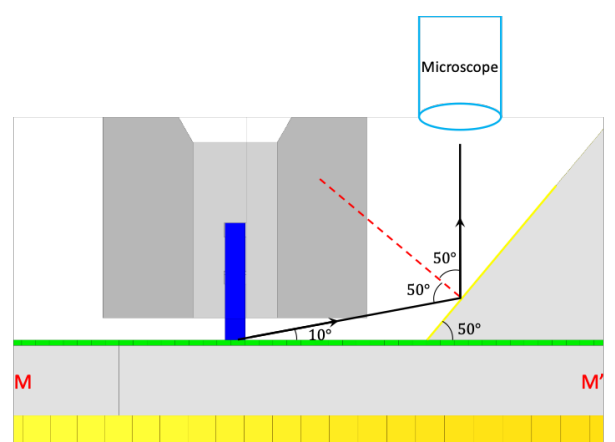

Fig. 14. Optical circuit of probe, mirror and microscope in side view from M-M' plane (not to scale).

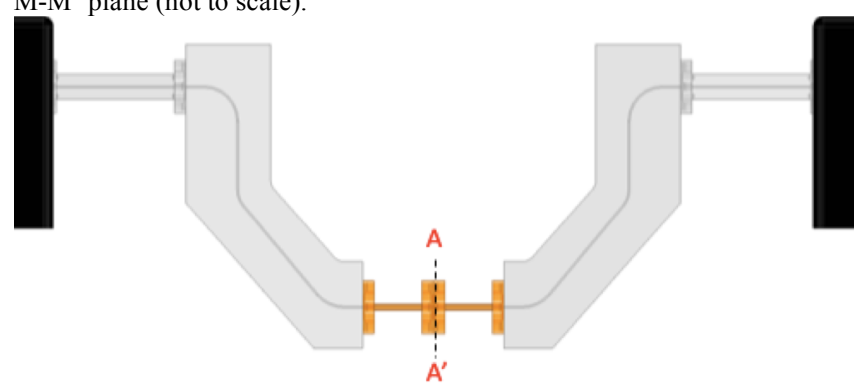

(a)

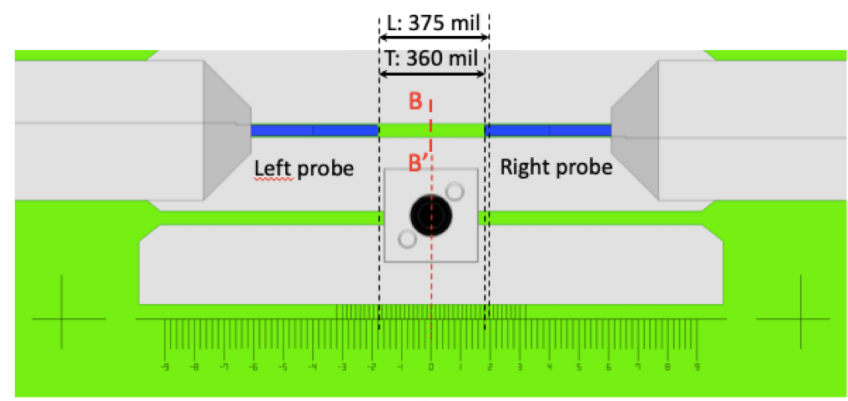

(b)

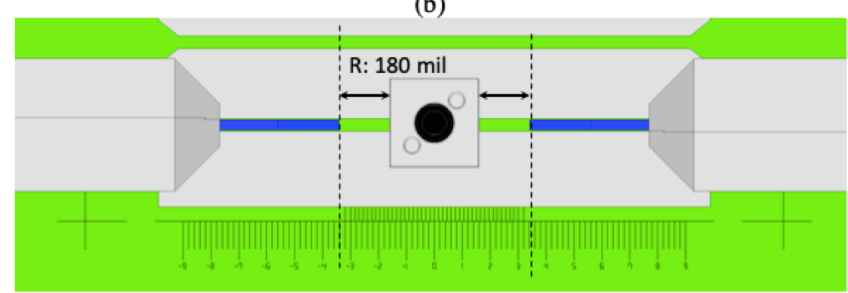

(c)

Fig. 15. Calibration Methods (a) WR 5.1 TRL kit for A-A' plane in side view, (b) SIIG-based T/L kit for B-B' plane, and (c) SIIG-based R kit in top view. 


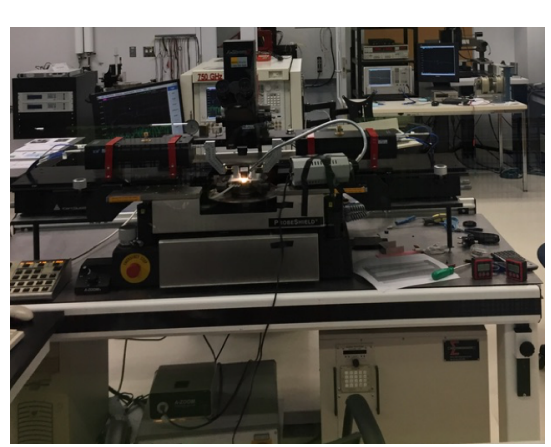

(a)

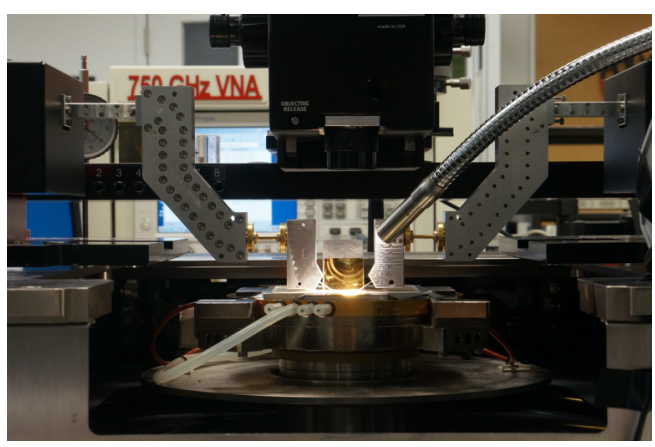

(b)

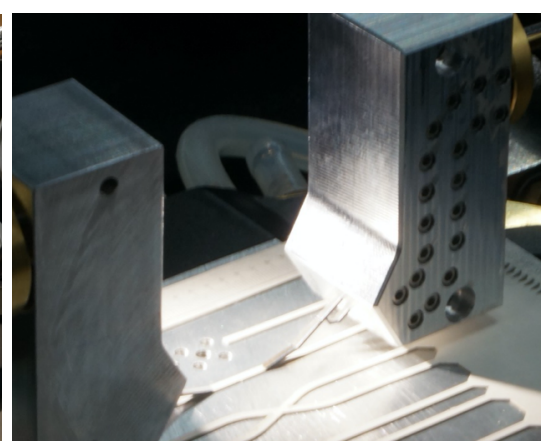

(c)

Fig. 16. (a) Whole measurement set up, (b) side view of measurement setup and (c) measurement setup of the 360 mil-length SIIG.

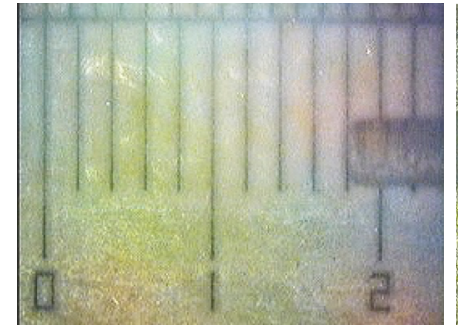

(a)

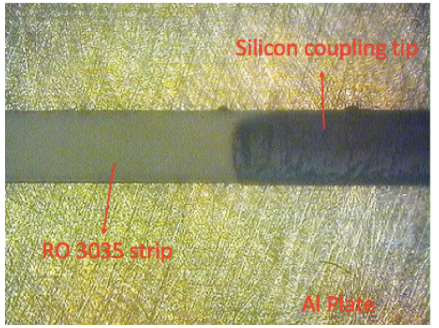

(b)

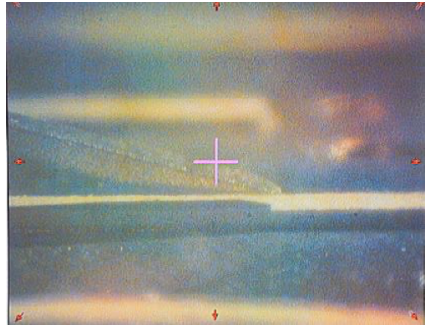

(c)

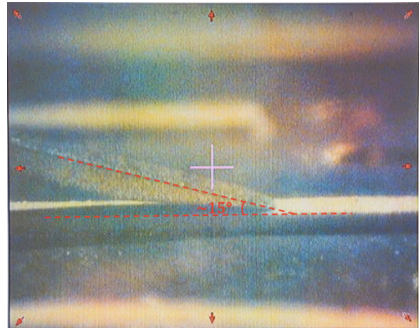

(d)

Fig. 17. Top view of probe with (a) alignment graduation markings and (b)SIIG. Side view of probe (c) align mode (200 $\mu$ m height distance) and (d) contact mode.

in Fig. 16(a). To do the probe contact, the side view of the silicon probe and the SIIG should be seen in detail. A $50^{\circ}$ tilt mirror was designed to reflect the side view into the microscope. The simple optical circuit is shown in Fig. 14.

\section{A. Experimental Procedure}

Step 1: Waveguide calibration. Set the measurement system as shown in Fig. 15(a) and calibrate it to the A-A' plane of the interface of the two CERNEX 1-inch straight waveguides with the TRL method. Then, connect the two silicon probes to the WR5.1 waveguide flanges as described in Fig. 16(b).

Step 2: Probe alignment and contact. First, as shown in Fig. 17 (a), the alignment graduation markings are applied to find the $\mathrm{x}$-axis location of the probe tip. Then, under "align mode" (200 $\mu \mathrm{m}$ height distance between the silicon probe tip and the SIIG surface), the probe is moved to the upward side of the SIIG sample under test. As shown in Fig. 17 (b), the silicon probe is on the middle of the dielectric strip of the SIIG, pictured by microscope from the top view. The probe under the align mode, pictured by microscope from the mirror reflection, is clearly shown in Fig. 17(c). Finally, under the contact mode, the tip of the probe is onto the surface of the SIIG, as shown in Fig. 17(d). Step 3: Measure with waveguide calibration. Use the probes to contact a 9.14-mm (360-mil) length SIIG, as shown in Fig. 16(c). Measure S-parameters.

Step 4: Probe TRL calibration. The measurement system needs to be calibrated to the B-B' plane, which is the middle of a 9.14-mm (360-mil) length SIIG, as shown in Fig. 15(b). For the thru standard, the two probes are respectively moved to $-4.57 \mathrm{~mm}(-180 \mathrm{mil})$ and $4.57 \mathrm{~mm}(180 \mathrm{mil})$ positions, as shown in Fig. 15(b). For the line standard, keep the left probe in $-4.57 \mathrm{~mm}(-180 \mathrm{mil})$ position and shift the right probe to 4.95 $\mathrm{mm}$ (195 mil) position. For the reflect standard, mount the short block onto the aluminum plate and shift the probes to $4.57 \mathrm{~mm}$ (180 mil) away of the block edge, as shown in Fig. 15(c). We
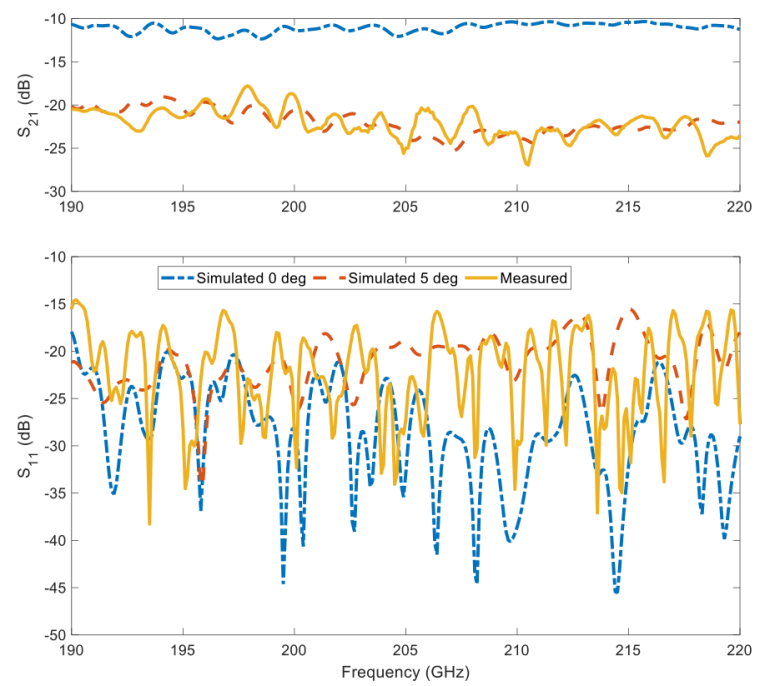

Fig. 18. Simulated and measured results of back-to-back silicon probes and 360-mil length SIIG. S-Parameters measured with the calibrated plane of A-A' plane.

used the Keysight PNA-X N5274A VNA to build the TRL calibration.

Step 5: Measure with probe calibration. Move two probes to $-12.7 \mathrm{~mm}(-500 \mathrm{mil})$ and $12.7 \mathrm{~mm}(500 \mathrm{mil})$ positions, respectively, and measure the S-parameters of the T/L SIIG, the coupler, the reference and the two adjacent SIIGs.

\section{B. Post-simulation and Measured Results}

As shown in Fig. 17(d), the intersection angle $\theta_{3}$ between the silicon probe tip and the SIIG top surface is approximately $5^{\circ}\left(\theta_{3} \approx 15^{\circ}-\theta_{1}=15^{\circ}-10^{\circ}=5^{\circ}\right)$, which affects the performance of the probe. Therefore, part of the EM energy will leak out into the air space from the intersection angle. A post-simulation based on $\theta_{3}=5^{\circ}$ was conducted. The post-simulated S-parameters of the back-to-back silicon probes 


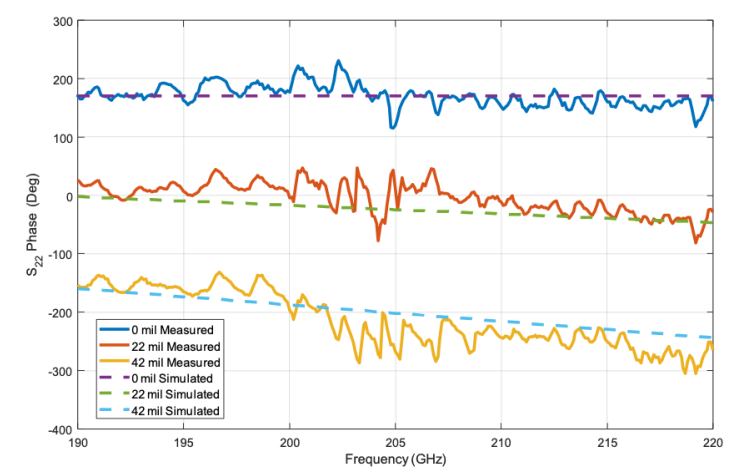

Fig. 19. Simulated and measured phases of offset short lines with calibrated silicon probes.

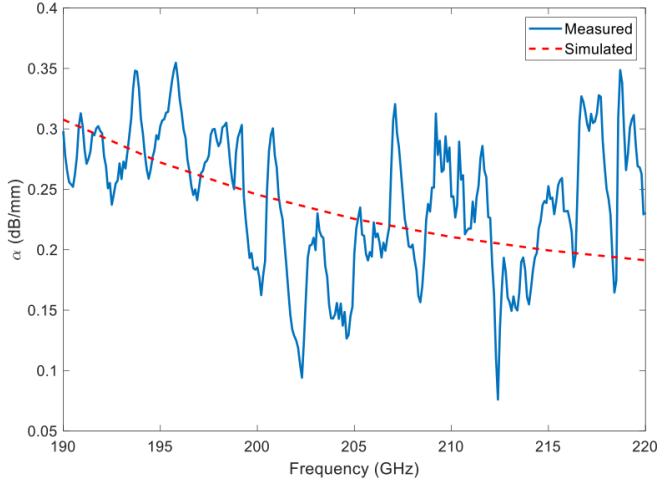

(a)

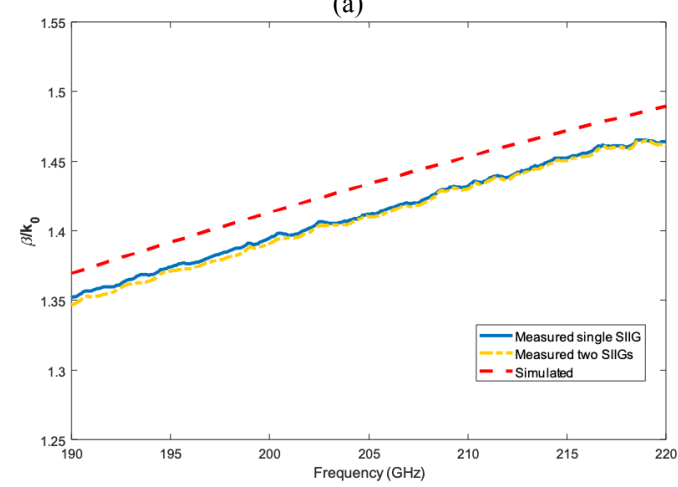

(b)

Fig. 20. Measured results of (a) attenuation constant and (b) normalized phase constant if the SIIG.

and the 9.14-mm (360-mil) length SIIG match well with the measured S-parameters of the Step 3, as shown in Fig. 18. The measured return loss of the silicon probe is better than $15 \mathrm{~dB}$. After removing the loss of the SIIG, the measured insertion loss of each probe is around $9.94 \mathrm{~dB}$. Compared with the ideal scenario $\theta_{3}=0^{\circ}$, the insertion loss is added approximately $5 \mathrm{~dB}$ for each silicon probe and SIIG. The reason of the intersection angle will be discussed in the next part. The measured and simulated phases of the offset short SIIG with different lengths are plotted in Fig. 19.

After the probe TRL calibration, the attenuation constant and the phase constant of the SIIG can be calculated by

$$
\left\{\begin{array}{c}
\alpha=-\frac{\left|S_{21}^{M}\right|}{L_{M}-L_{T}} \\
\beta=-\frac{\angle S_{21}^{M}-2 \pi \cdot N}{L_{M}-L_{T}},
\end{array}\right.
$$

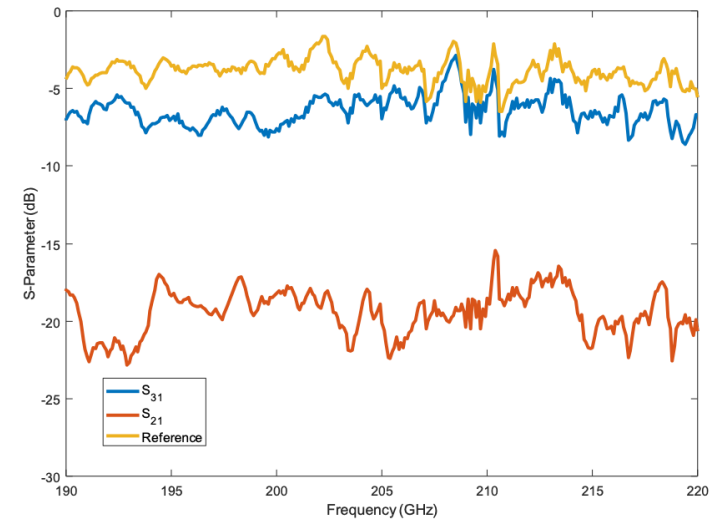

(a)

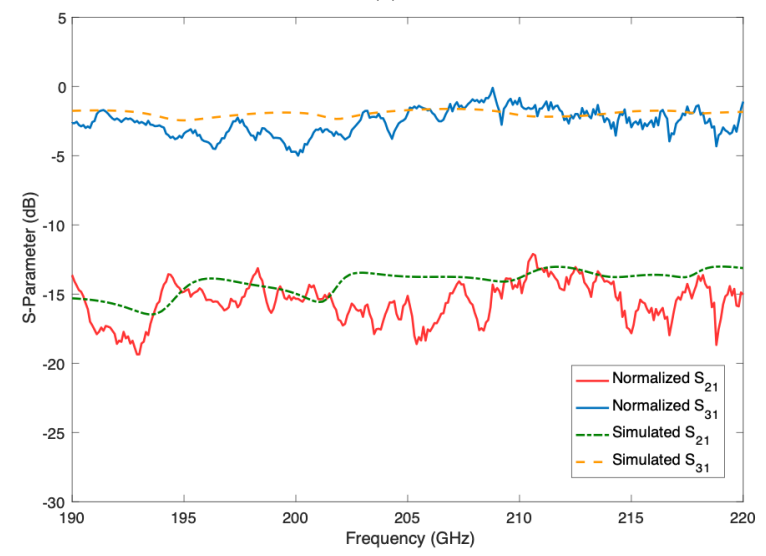

(b)

Fig. 21. (a) Measured results of the coupler and (b) normalized results of the coupler.

where $\left|S_{21}^{M}\right|$ and $\angle S_{21}^{M}$ are the magnitude and phase of $S_{21}$ of the measured SIIG, respectively; $L_{M}$ is the length of the measured SIIG; $L_{T}$ is the length of the thru of the SIIG TRL kit; and $N$ is the minima integer of $\left(L_{M}-L_{T}\right) / \lambda_{g}$. Here, $L_{M}$ and $L_{T}$ are $25.40 \mathrm{~mm}(1000 \mathrm{mil})$ and $9.14 \mathrm{~mm}$ (360 mil), respectively. The measured attenuation constant and phase constant of the SIIG are plotted in Figs. 20(a) and (b), respectively. The measured attenuation constant is $0.241 \pm$ $0.057 \mathrm{~dB} / \mathrm{mm}$ over the range of $190-220 \mathrm{GHz}$. The measured normalized phase constant ranges between 1.35 and 1.46 , which is lower than its simulated counterpart with $1.5 \%$ relative difference. The reason for this difference is related to the substrate in the SIIG, and the substrate in the measurement of permittivity comes from a different batch of the substrate RO3035 from Rogers. As shown in Fig. 20(b), the measured normalized phase constant of the two adjacent SIIGs from port 3 to port 4 is almost the same as the single SIIG results. Therefore, there is almost no coupling between the two adjacent SIIGs with a $2.54 \mathrm{~mm}$ distance.

The reference SIIG and SIIG-based coupler were also measured. The parameter $S_{31}$ of the coupler measurement setup is shown in Fig. 13. The SIIG-based coupler through port 1 was excited with the left silicon probe, and the energy of port 3 is coupled to the right silicon probe. Meanwhile, port 2 and port 4 are matched by the absorber directly. The measured results of the reference and coupler are shown in Fig. 21(a). The reference structure can be used to calculate the normalized $S_{21}$, as follows, 


$$
\text { Normalized } S_{i 1}(d B)=S_{i 1}(d B)-S_{21, \text { Ref. }}(d B) \text {, }
$$

where $S_{i 1}$ is the measured result of the coupler $(i=2,3)$, and $S_{21, R}$ is $S_{21}$ of the reference SIIG. As shown in Fig. 21(b), the normalized results and the simulated results basically agree with each other.

\section{Error Analysis}

The random error and the systematic error are the two main sources of error in the measurements.

The random error is caused by the alignment uncertainty. We align the probe position using the alignment graduation markings, as shown in Fig. 17(a). The maximum line width of the fabricated alignment graduation markings is $53.17 \mu \mathrm{m}$, which indicates the alignment uncertainty is $26.6 \mu \mathrm{m}$. Considering the propagation constant of the SIIG in Fig. 20, the magnitude uncertainty and phase uncertainty are $0.008 \mathrm{~dB}$ and $9^{\circ}$, respectively.

For the systematic error, the entire probe measurement system is placed on the Suss probing station. After setting all the components of the measurement system, the intersection angle exists $\left(\theta_{3}=5^{\circ}\right)$ between the silicon probe tip and the SIIG top surface. The intersection angle is the systematic error, because the probing station cannot tune its elevation angle. Moreover, there is a $0.3^{\circ}$ mechanical vibration which is caused by the cooling fan of the VDI WR5.1 extender. The vibration direction is along the y axis in Fig. 13. To evaluate the effect of the intersection angle, we studied the relationship between $\theta_{3}$ and the error box. The signal flow graph of back-to-back error boxes is shown in Fig. 22(a). The structure model and the full-wave EM simulated results (direct simulated results) of the error are shown in Fig. 10(a). We also performed the full-wave EM simulation of the TRL model shown in Fig. 15, and carried out the TRL calibration. The calibrated results are called the "TRL simulated results". When $\theta_{3}$ is equal to $0^{\circ}$, the TRL simulated and direct simulated $S_{11}$ and $S_{21}$ of the error box are matched well, as shown in Fig. 22(b). The TRL simulated and direct simulated $S_{22}$ of the error box are generally matched. When $\theta_{3}$ is equal to $5^{\circ}$, the measured, TRL simulated and direct simulated $S_{11}$ and $S_{21}$ of the error box are generally matched, as shown in Fig. 22(c). However, the measured and TRL simulated $S_{22}$ do not match with the direct simulated $S_{22}$. The reason is that the coupling tip is sensitive to the change of $\theta_{3}$, as shown in Fig. 3. Therefore, when $\theta_{3}$ is equal to $5^{\circ}$, the silicon probe can only characterize $S_{21}$ of the device under test.

To eliminate the intersection angle, the best method is mounting the VDI WR5.1 extender onto an inclined surface, which can tune its elevation angle. Another solution is to use the mini VDI WR5.1 extenders.

\section{Discussions}

Compared with the contact type GSG probe, such as the GGB probe [11] and micro-machined probe [12], the proposed silicon probe does not need an extra transition between the GSG probe and the dielectric waveguide under measured. Compared with the non-contact type probe, such as the slot array coupling-based probe [19], the quasi-optical path and planar antenna-based probe [20], and the H-plane dielectric horn-based probe [5], the proposed probe does not need to design any

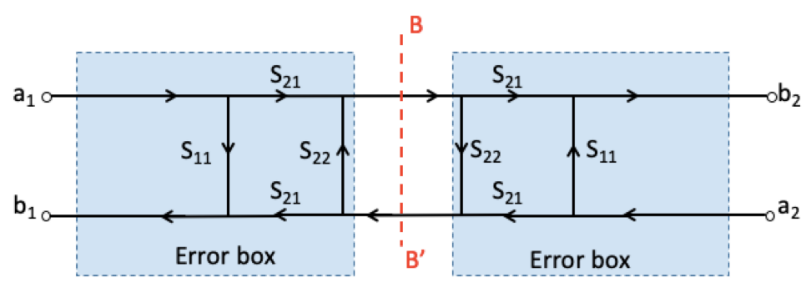

(a)

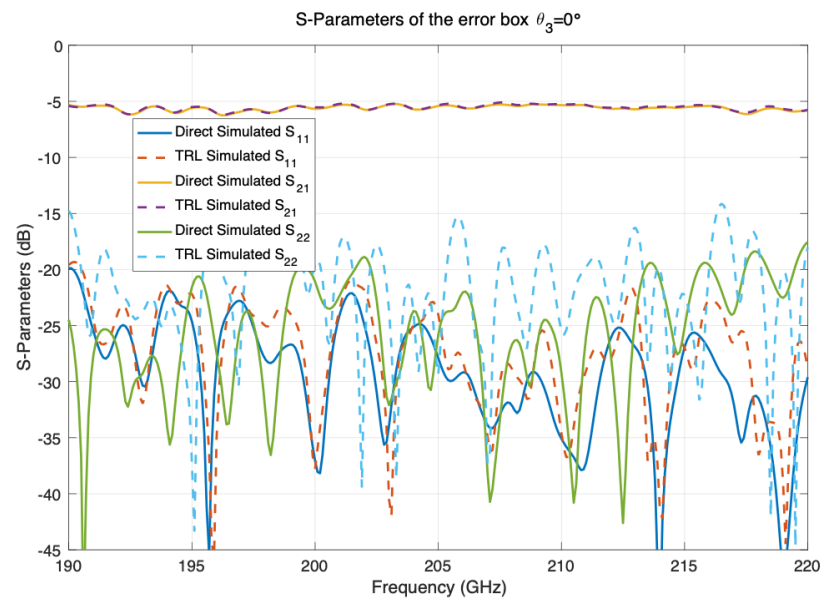

(b)

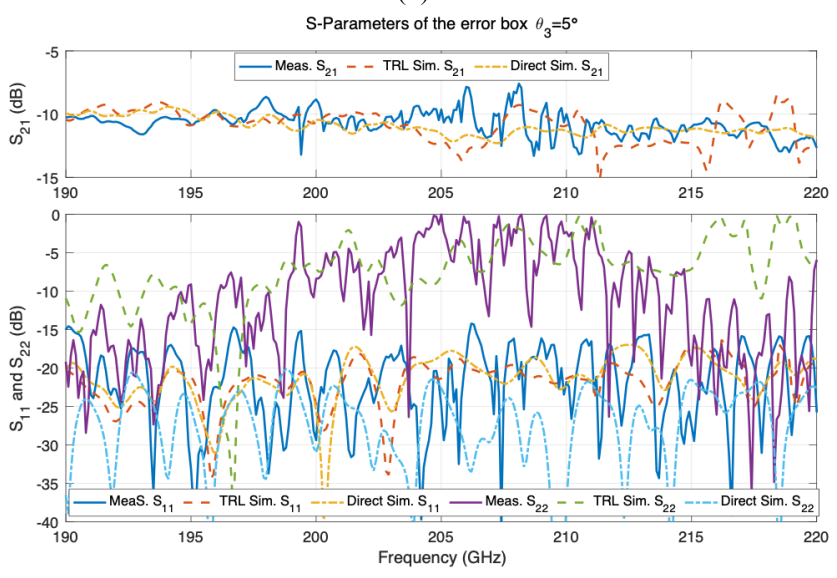

(c)

Fig. 22. S-parameter of error box. (a) Signal flow graph of the thru for TRL calibration. (b) TRL simulated and direct simulated results at $\theta_{3}=0^{\circ}$. (c) Measured results, TRL simulated results and direct simulated results at $\theta_{3}=5^{\circ}$.

antenna or quasi-optical path. The probes of [11], [12] and [20] have an excellent measurement accuracy.

\section{CONCLUSION}

We introduced and presented a dielectric probe technique concept for measuring and characterizing dielectric waveguide and its components in 190-220 GHz. The work principle of the probe is explained in this paper. The silicon probes and waveguide blocks were successfully fabricated and assembled. The mirror was applied to see the side view of the silicon probe and SIIG under a microscope. The propagation constant of the SIIG was successfully characterized over the band of 190-220 $\mathrm{GHz}$. The measured results of the SIIG-based coupler are presented, and they agreed with simulated results. Future work will be set to develop higher frequency prototypes, improve 
probe performances, and apply the probe to feed a SIIG-based leaky-wave antenna array.

\section{ACKNOWLEDGEMENT}

The authors wish to acknowledge the technical support of several staff members in the Poly-Grames Research Center: Dr. David Dousset, Steve Dube and Traian Antonescu. The authors would also like to thank the Rogers Corporation for providing free samples for fabricating the SIIG.

\section{REFERENCES}

[1] C.-S. Shi et al., "Terahertz and far-infrared windows opened at Dome A in Antarctica". Nat. Astron. vol. 1, 0001, 2016.

[2] M. C. Wiedner et al., "A proposed heterodyne receiver for the origins space telscope," IEEE Trans. THz Sci. Technol.,vol. 8, no. 6, pp. 558-571, Nov. 2018.

[3] P. F. Goldsmith and D. C. Lis, "Early science results from the heterodyne instrument for the far infrared (HIFI) on the Herschel space observatory," IEEE Trans. THz Sci. Technol.,vol. 2, no. 4, pp. 383-392, Mar. 2012.

[4] T. Reck et al., "A silicon micromachined eight-pixel transceiver array for submillimeter-wave radar," IEEE Trans. THz Sci. Technol.,vol. 5, no. 2, pp. 191-206, Mar. 2015.

[5] H. T. Zhu and Q. Xue, "Determination of propagation constant of terahertz dielectric ridge waveguide using non-contact measurement approach," IEEE Trans. Instrum. Meas. vol. 66, no. 8, pp. 2118-2128, Aug. 2017.

[6] H. T. Zhu et al., "Design, fabrication and measurement of the low-loss SOI-based dielectric microstrip line and its components," IEEE Trans. THz Sci. Technol., vol. 6, no. 5, pp. 696-705, Sep. 2016.

[7] H. T. Zhu et al., "Low-cost narrowed dielectric microstrip line- a three dielectric waveguide based on PCB technology for millimeter-wave applications," IEEE Trans. Microw. Theory Tech., vol. 65, no. 1, pp. 119-127, Jan. 2017.

[8] A, Patrovsky and K. Wu, "Substrate integrated image guide (SIIG) - a planar dielectric waveguide technology for millimeter-wave applications," IEEE Trans. Microw. Theory Tech., vol. 54, no. 6, pp. 2872-2879, Jun. 2006.

[9] N. Ranjkesh et al., "1.1 THz U-silicon-on-glass (U-SOG) waveguide: a low-loss platform for THz high-density integrated circuits," IEEE Trans. THz Sci. Technol., vol. 8, no. 6, pp. 702-709, Nov. 2018.

[10] P. F. Goldsmith, Quasioptical Systems: Gaussian Beam Quasioptical, Propagation and Applications, 1st ed. New York, NY, USA: IEEE Press, 1998.

[11] GGB Industries Inc. GSG Model 220 http://www.ggb.com/PdfIndex files/mod220.pdf

[12] T. J. Reck et al., "Micromachined probes for submillimeter-wave on-wafer measurements - part I: mechanical design and characterization," IEEE Trans. THz Sci. Technol., vol. 1, no. 2, pp. 349-356, Nov. 2011.

[13] T. J. Reck et al., "Micromachined probes for submillimeter-wave on-wafer measurements- part II: RF design and characterization," IEEE Trans. THz Sci. Technol., vol. 1, no. 2, pp. 357-363, Nov. 2011.

[14] N. Dolatsha and J. Hesselbarth, "Millimeter-wave chip-to-chip transmission using an insulated image guide excited by an on-chip dipole antenna at $90 \mathrm{GHz}$," IEEE Microw. Wireless Compon. Lett., vol. 22, no. 5, pp. 266-268, May 2012.

[15] A. Patrovsky and K.Wu, "94-GHz broadband transition from coplanar waveguide to substrate integrated image guide (SIIG)," in Proc. 2007, IEEE MTT-S Int. Microwave Symp. Dig., pp. 1551-1554.

[16] B. Yu et al, "Ring-resonator-based sub-THz dielectric sensor," IEEE Microw. Wireless Compon. Lett., vol. 28, no. 11, pp. 969-971, Nov. 2018.

[17] H. T. Zhu and K. Wu, "Complex permittivity measurement of dielectric substrate in sub-THz range," IEEE Trans. THz Sci. Technol., to be published.

[18] D. M. Pozar, Microwave Engineering, 3rd ed. NewYork: JOHN WILEY \& SONS, INC, 2005.

[19] M. Moallem and K. Sarabandi, "A non-contact submillimeter-wave S-parameters measurement technique for multiport micro-machined devices," IEEE Trans. THz Sci. Technol., vol. 4, no. 3, pp. 338-346, May 2014.

[20] C. Caglayan, G. C. Trichopoulos and K. Sertel, "Non-contact probes for on-wafer characterization of sub-millimeter-wave devices and integrated circuits," IEEE Trans. Microw. Theory Tech., vol. 62, no. 11, pp. 2791-2801, Nov. 2014.

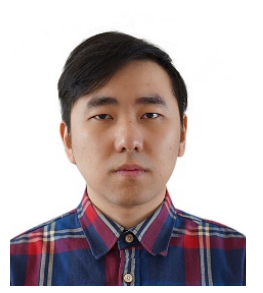

Hao-Tian Zhu (S'12-M'17) was born in Nanjing, China, in 1989. He received the B.S. degree in electronic engineering from the Nanjing University of Science and Technology, Nanjing, China, in 2011, and Ph.D degree in electronic engineering from the City University of Hong Kong, Kowloon, Hong Kong, in 2016.

He was a Visiting Scholar with the Purple Mountain Observatory, Chinese Academy of Sciences, Nanjing, in 2016. He was a Postdoctoral Fellow with State Key Laboratory of Millimeter Waves, City University of Hong Kong and the Polytechnique de Montréal, Montréal, QC, Canada in 2016-2017 and 2017-2018, respectively. He was a Postdoctoral Fellow with 1'Observatoire de Paris, University PSL, CNRS, LERMA, Paris, France in 2018-2020. His research interests include THz dielectric transmission lines, antenna, $\mathrm{THz}$ micromachining fabrication and assembling technology, dielectric substrate permittivity measurement in $\mathrm{THz}$ range, and $\mathrm{THz}$ antenna measurement system.

Dr. Zhu was a recipient of the Best Student Paper Award at the 2013 IEEE International Workshop on Electromagnetics and the Champion of Student Paper Competition in MTT Section of the IEEE Hong Kong AP/MTT Joint Postgraduate Conference in 2015. He was selected as a Finalist in the student paper competition of the 2015 IEEE MTT-S International Microwave Symposium.

Jules Gauthier, photograph and biography not available at the time of publication

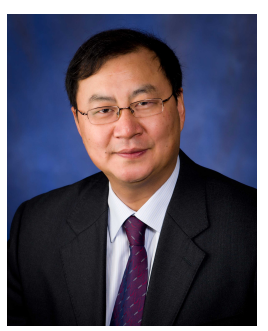

Ke Wu (M'87-SM'92-F'01) received the B.Sc. degree (with distinction) in radio engineering from the Nanjing Institute of Technology (now Southeast University), China, in 1982 and the D.E.A. and Ph.D. degrees in optics, optoelectronics, and microwave engineering (with distinction) from the Institut National Polytechnique de Grenoble (INPG) and University of Grenoble, France, in 1984 and 1987, respectively.

$\mathrm{Dr}$. Wu is a Professor of electrical engineering, and endowed Industrial Research Chair in Future Wireless Technologies with the Polytechnique Montréal (University of Montreal), QC, Canada. He has been the Director of the Poly-Grames Research Center. He was the founding Director of the Center for Radiofrequency Electronics Research of Quebec (Regroupement stratégique of FRQNT) and Tier-I Canada Research Chair in $\mathrm{RF}$ and millimeter-wave engineering. He is also an adjunct professor with the School of Information Science and Engineering, Ningbo University. He has held guest, visiting, and honorary professorships with many universities around the world. He has authored or co-authored over 1300 referred papers and a number of books/book chapters. He has filed more than 50 patents. His current research interests involve substrate integrated circuits and systems, antenna arrays, field theory and joint field/circuit modeling, ultra-fast interconnects, wireless power transfer and harvesting, and MHz-through-THz technologies and transceivers for wireless sensors and systems as well as biomedical applications. He is also interested in the modeling and design of microwave and terahertz photonic circuits and systems.

Dr. Wu is a Fellow of the Canadian Academy of Engineering (CAE) and of the Royal Society of Canada (The Canadian Academy of the Sciences and Humanities). He is a Member of Electromagnetics Academy, Sigma Xi, URSI, and IEEE-Eta Kappa $\mathrm{Nu}$ (IEEE-HKN). He has held key positions in and has served on various panels and international committees including the chair of Technical Program Committees, International Steering Committees, and international conferences/symposia. In particular, he was the General Chair of the 2012 IEEE Microwave Theory and Techniques (IEEE MTT-S) International Microwave Symposium (IMS). $\mathrm{He}$ has served on the Editorial/Review Boards of many technical journals, transactions, proceedings and letters as well as scientific encyclopedia including Editor and Guest Editor. He was the Chair of the joint IEEE Montreal chapters of 
MTT-S/AP-S/LEOS and then the restructured IEEE MTT-S Montreal Chapter Canada. He has served IEEE MTT-S and Administrative Committee (AdCom) as Chair of the IEEE MTT-S Transnational Committee, Member and Geographic Activities (MGA) Committee, Technical Coordinating Committee (TCC) and 2016 IEEE MTT-S President among many other AdCom functions. Currently, he is the Chair of the IEEE MTT-S Inter-Society Committee. He was an IEEE MTT-S Distinguished Microwave Lecturer (2009-2011). Dr. Wu was the inaugural representative of North America as a Member of the European Microwave Association (EuMA) General Assembly. He was the recipient of many awards and prizes including the first IEEE MTT-S Outstanding Young Engineer Award, the 2004 Fessenden Medal of the IEEE Canada, the 2009 Thomas W. Eadie Medal of the Royal Society of Canada, the Queen Elizabeth II Diamond Jubilee Medal in 2013, the 2013 FCCP Education Foundation Award of Merit, the 2014 IEEE MTT-S Microwave Application Award, the 2014 Marie-Victorin Prize (Prix du Quebec - the highest distinction of Québec in the natural sciences and engineering), the 2015 Prix d'Excellence en Recherche et Innovation of Polytechnique Montréal, the 2015 IEEE Montreal Section Gold Medal of Achievement, and the 2019 IEEE MTT-S Microwave Prize. 